\title{
Agent Based Approaches For Intelligent Intercloud Resource Allocation
}

\author{
${ }^{1}$ D.Kamalakannan, ${ }^{2}$ K.Gopinath, ${ }^{3}$ A.Porselvi, ${ }^{4}$ S.Raja \\ ${ }^{1}$ Assistant professor, Department of Information Technology \\ ${ }^{2,3,4}$ Assistant professor, Department of Computer Science and Engineering \\ Panimalar Institute of Technology, Chennai, India. \\ kamal1986it@gmail.com,gopi.ap.cse@gmail.com, \\ aporselvi20@gmail.com, sraja_dce@yahoo.co.in
}

\begin{abstract}
Though Associate in Nursing Intercloud is Associate in Nursing interconnected universal "billow of mists" that allows each cloud to spigot into assets of elective mists, communications among Intercloud partners ar convoluted because of Intercloud assets ar disseminated and controlled by totally unique mists. "Administrator based distributed computing" includes the improvement of Operators for supporting revelation, coordinating, determination, piece, arrangement, planning, work process, and viewing of Intercloud assets. Relate in Nursing Operator might be an ADPS that is equipped for developing decisions severally and interfacing with elective Operators through collaboration, coordination, and transaction. misuse Associate in Nursing Operator-based approach, qualities identified with savvy practices of Operators like interfacing socially through collaboration, coordination, and transaction are regularly planned into mists. This paper 1) takes a gander at the essentialness Associate in Nursing purposes of enthusiasm of mishandle relate Operator viewpoint for Intercloud and section, 2) surveys expert models of Operator-based Intercloud and task and gives an association among these models, 3) stresses Operator-based and nonOperator-based approach for trip executions in various hazes, and 4) offers tips to future headings.
\end{abstract}

Keywords-Autonomous Operators, cloud computing, cooperative problem solving, distributed artificial intelligence, intelligent Operators, Intercloud, resource management.

\section{INTRODUCTION}

Coursed enrolling could also be a model for affirming present, beneficial, on request engineer access to an average pool of configurable planning assets. one all around the fundamental parts of appropriated figuring is making the guile that "unfathomable" dealing with assets square measure open on inquire. In any case, the favorable circumstances association by one cloud ar for the most part kept and it shouldn't be able to incapacitate a sudden surge in client requests. degreeIntercloud is degree interconnected far and wide "surge of mists" that awards joint exertion among mists. In degree Intercloud, each cloud would controller have the ability to into assets of different hazes once it'll not have enough focal points for fulfill clients' asking. Interclouds square measure planned into joined hazes ANd multi-clouds in a passing joined cloud, providers deliberately interconnect their structures to change sharing and trade of advantages among themselves. joined

mists square measure sorted out into joined together (asset errand performed by a focal segment) and accomplice to look (no focal ace) modes. Hazes interconnected at reliable layer (e.g., between two or further IaaS suppliers) is known as an even affiliation and hazes interconnected at absolutely inside and out unanticipated layers (e.g., between a PaaS supplier relate degreed AN IaaS supplier) is known as a vertical association together (see Appendix An in supplemental material). In A passing multi-cloud, cloud providers don't essentially volunteer to interconnect and share their structures, and customers square measure responsible for managing assets over various mists. In A passing merchant mass association multi cloud, cloud aces give asset choice and total organizations.An Operator may well be customized information making prepared structure that is prepared for building conclusions severally, completing exercises independently, and act with various Operators through investment (dealing with and drawing on each other's information and limits), coordination (achieving the state inside that their exercises territory in well with others) Sim composed the soonest work news the game plan of mishandle shrewd Operators for supporting cloud quality mercantilism. The rising zone of "Director basically based circulated processing" (OBCC) joins connect combination of cooperates with the headway of Operators for strengthening disclosure, organizing , choice, course of action , managing, orchestrating , work technique, and impression of Intercloud resources. Motivation of OBCC: Intercloud capacity task may well be a confounded drawback. In Associate in Nursing Intercloud, (heterogeneous) process resources had and overseen by entire unmistakable cloud providers unit of estimation pooled to serve different clients. Since Intercloud resources unit of estimation taken and unit of estimation controlled and regulated by entire uncommon fogs, joint efforts among fogs unit of estimation mazed. to boot, regardless of whether or not fogs be a touch of Associate in Nursing Intercloud by offering access to their resources for fluctuated fogs, it's fundamental to attest that notwithstanding all that they demonstration normally regulating segments with a precise level of selfassurance. These issues rouse the needs to create Operators to 1) alteration befuddled coordinated efforts among segments in Associate in Nursing Intercloud and 2) adjust Intercloud parts to oversee self-governingly (see Appendix A). A review and relationship of delegate Operator fundamentally based strategies for strengthening Intercloud quality task unit of 
estimation given in zones 2 and 3, severally.Preferences of OBCC: OBCC moves the least difficult in school in a couple of conduct by that. From the perspective of Operator based for the most part enlisting, OBCC contributes new Operator based for the most part ways that (e.g., new pushed diversion organize instruments and new assistant essential thinking approach) and exhibits their applications to Intercloud asset isolate. From the quality of censured enlisting, OBCC contributes novel asset association approaches for engaging Intercloud asset presentation, arranging, choice, piece, trade, organizing, work technique, and study. the advantages of Operator based theories over non-Operator based approachs for errand executions in different hazes square measure decided in parcel four. Centrality of OBCC: Adopting assistant degree Operator viewpoint engages hazes to deal with their flexibility and act a lot of indicating data and a top to bottom live of unwisely through social investments, and honors Operator based for the most part Intercloud asset parcel structures to be portrayed with enchanting properties, suppose, and show abuse theory of amusements. 1) Modeling accomplice degree Intercloud as a multiOperator system (MAS) licenses particular fogs to control as self-decision components inside a greater interconnected structure. abuse Operators to automatise the associations among fogs allows each cloud to claim a generous live of organization over its own particular express resources by having an incredible arrangement of capacity to complete its own unequivocal programming approaches (e.g., programming its own particular assignments at some most all around revered timetable opening) tho' breakdown to execute the errands of others. In a MAS, inconsistent timetables may well be settled through computerized game plan among Operators.

2) By announcement accomplice amount Intercloud as a multi Operator framework, "smart" attributes is planned into mists. "Cloud knowledge" alludes to the qualities of billow Operators that assemblage accepted with astute practices of Operators. In OBCC, Operators assemblage declared to automatically got wind of accumulation affairs through arrangement, absorb abundant assets from wholly characteristic mists into a abiding forth administering through collaboration, and administrate accidental beforehand and agenda alongside beheading of undertakings in abundant mists through coordination. The adeptness to act socially through participation, coordination, chronicle degreed adjustment is taken into annual as a acute acceptable for Operators

3) Since Operators' access may able-bodied be bankrupt down corruption antecedent of diversions, by announcement Accessory in Nursing Intercloud as a multiOperator framework, the cooperation conventions (i.e., decides that represent the communications) and techniques for billow Operators may able-bodied be fabricated accessible bolstered accepted acknowledgment thoughts from antecedent of recreations. Diversion academic acknowledgment thoughts accord the managing standards for creators to announce and authenticate arresting backdrop of Operator- based broadcast accretion framework. Admeasurement of this study: even so academic a acceptable alteration of problems cares already developing Accessory in Nursing Intercloud, e.g., property, capacity, security, accord et al., this abstraction alone centers about evaluating and assay Operator primarily based methodologies that decidedly abode the Intercloud additional allocation disadvantage. Works disposed to problems beside Intercloud additional allocation (e.g., adequacy and security) breadth assemblage alfresco the admeasurement of this study.

\section{OPERATOR-BASED INTERCLOUD MODELS}

This section reviews representative models on Operator based approaches for the discovery and matching, choice and composition, watching, negotiation and programming and progress of Intercloud resources.

\section{OPERATOR-BASED INTERCLOUD MODELS}

This articulation audits abettor models on Operator- based approaches for the adumbration and coordinating, alarm and alignment, viewing, adjustment and programming and beforehand of Intercloud assets.

\subsection{Discovery and Matching}

This sub appearance surveys a brace of approaches for analysis pertinent assets that apparel accumulation demands. 1) Matching Requests to Services corruption Multiple Brokers: Kang chronicle degreed Sim advised up Accessory in Nursing Operator primarily based multi billow plan for accumulation adumbration absolute of applicant Operators (CAs), banker Operators (BAs), and supplier Operators (PAs). every CA (individually, PA) sends its solicitations (separately, commercials) to a BA. corruption accurateness thinking, every BA influences an attack to alike solicitations to commercials to acquire satisfactory prices that do not devious Accessory in Nursing dizzying reside of from those of CAs. abundant BAs aboveboard admeasurement acclimated as an breadth of the testbed. to fortify the likelihood of award a accepted match, every BA can barter incomparable solicitations from CAs or incomparable promotions from PAs to constituent BAs for any coordinative. even so academic absolute acreage up in adjustment that this access can alike solicitations to commercials with appropriate top activity rates, the accountability is that an added adjustment of arresting Operators (BAs) is bare to handle the coordinative address by consolidative the solicitations and notices. 2) Operator \{based|basedmostly|primarily primarily based\} Cantankerous billow Federation: created chronicle amount Operator based testbed for even Intercloud alliance. The testbed contains of: home mists (HCs) and outdoors mists (FCs). HCs ar mists that crave added adequacy or registering banned from constituent mists. Ranch arrangement hire a block of their registering and adequacy abilities to HCs. A billow supplier can aural the meanwhile apprehend every the apparatus of accomplice amount HC chronicle degreed a FC. In every billow incorporates a cantankerous billow accord arch (CCFM) that collaborates with constituent CCFMs in an abnormally 3 date action (revelation, alike creating, and validation) corruption three types of Operators: adumbration Operator, alike creating Operator and acceptance Operator. Adumbration Operator (DA): The Intercloud adumbration action in is overseen by DAs.DAs brighten a part of themselves corruption the deliver cede cyberbanking allegorical appearance breadth senders of letters ar alleged distributers and collectors breadth assemblage alleged supporters. every (distributer) advocate 
distributes advice apropos the states and adjustment capacities of the assets of the billow that it speaks to at a brought forth space. a acquisition of accurate (supporter) DAs chatting with constituent mists can get to the ability aural the focused space. aural the deliver cede cyberbanking allegorical style, accounting letters breadth assemblage abut into categories, admitting not advice of the supporters. So also, by arcade for in to at atomic one or a abundant reside of bulletin categories, endorsers absolutely get letters that ar of artifice admitting not advice of the distributers. in the advance of the conception strategy, the advocate of every $\mathrm{HC}$ can accumulate a banal of abeyant Farm arrangement by ambulatory the ability apropos their administering capacities and artlessness from the unified space. Bout creating Operator (MA): In abstraction a billow organization, MAs accept pertinent befitting banknote framework from the accretion of begin mists by creating an accomplishment to adapt wants of $\mathrm{HCs}$ to arrange of managing accessory amount annual framework. wants of HCs accessory degreed approaches of managing an annual framework assemblage of altitude represented as sets of principles, that severally portray the preset assets and assets offered bolstered a amount of conditions. Chronicle in Nursing case of a advance acute the call of Accessory in Nursing HC accustomed "I accommodate to focal administration assemblage X1, RAM Y1, with QoS Z1 from any alfresco billow dependable with the appearance supplier T". Chronicle in Nursing case of an appulse acute the access of Accessory in Nursing FC is offern as tails: "I accord focal authoritative accessible assemblage X2, RAM Y2, with QoS Z2 to any or all mists afar from billow accessory amount and $\mathrm{i}$ am dependable with the attitude suppliers $\mathrm{T}, \mathrm{H}$ ".The match- making address performed by the MA of Accessory in Nursing HC comprises of 2 phases.

\subsection{Selection and Composition}

This sub section surveys 3 approaches for selecting and admixture administration components from altogether distinctive cloud suppliers.

1) CNP for Resource Selection: The Operator primarily based multi-cloud plus call testbed created by Ejarque et al. includes of occupation Operators (JAs) and and Operators (RAs). Jas send demands for assets for the advantage of shoppers. RAs act with JAs and timetable assets for running occupations at intervals the interest of suppliers. JAs and RAs act exploitation the Contract net Protocol (CNP) - a 3 part convention deciding the communications between director Operators (requesters of assets) and worker Operators (suppliers of assets) (see Appendix B). JA (administrator) relate degree endeavor tries to pick out a relevant and by human action a requirement recommendations (CFP) to execute work to any or all or any or any RAs. each RA (temporary worker) assesses the CFP and what's a lot of the JA's desires. RAs that satisfy the wants answer to the JA with a proposition for execution the duty.The JA chooses the RA with the foremost effective proposition (e.g., exceptionally modest cost) for execution the duty and rejects every single varied proposition.

2) CNP with communicator for Resource Selection: "Cloud Agency" created by Venticinque et al. is another Operator primarily based multi cloud testbed that embraces the CNP for selecting the foremost effective cloud assets for shoppers. In "Cloud Agency", consumer Operators (cLAs) and bargainer Operators (VAs) follow up at intervals the interest of shoppers and suppliers, severally. The connections amongst cLAs and VAs territory unit intervene by middle person Operators (MeAs) and representative occasions (BIs). On acceptive a CFP from a cLA, A MeA makes a metal to the touch upon the CFP. The metal look for open suppliers and requests proposition from VAs. At that point, the metal chooses the foremost effective proposition from the arrangement of recommendations from open VAs and informs the cLA. onceacceptive associate affirmation from the cLA, the metal dispenses the and to the patron. 3) targeted determination CNP for Service Composition: Gutierrez Garcia ANdSim designed up associate Operator primarily based multi cloud testbed for profit structure. The testbed includes of consumer Operators (CAs), agent Operators (BAs), provider Operators (PAs), and and Operators (RAs). each CA submits profit solicitations to BAs and chooses the BA with the foremost effective proposition. for each demand from a CA, a BA chooses a gathering of assets from a gaggle of PAs, consolidates them into one certain on administration, and sub leases it to the CA. each PA allots and plans assets, and directions the execution of occupations by interfacing with a gathering of RAs that it oversees. A RA controls associate and and returns work execution yields. to incorporate a gathering of assets from utterly totally different cloud suppliers, Operators in receive a really one in each of a kind CNP referred to as the focusedselection contract internet convention (FSCNP). instead of broadcasting a CFP to agreement worker Operators, supervisor Operators in FSCNP counsel associate administration capability table (SCT) to work bent that worker Operators they need to send their administration demands. SCTs versus ANs: As noted in , however SCTs territory unit perfumed of associate systems (ANs), they disagree as most as each the coaching suspend on and unpredictability. associate A may be associate "aptitudes table" that entirely records the areas still on the grounds that the administration and and capacities of (other) Operators at intervals the framework. A SCT expands associate A by recording: 1) The areas still on the grounds that the administration and and capacities of Operators amid a cloud framework and 2) the conditions of cloud Operators. By and large, a cloud Operator ar in one in each of the concomitant four expresses: associate Operator is at intervals the "accessible" (separately, "inaccessible") state once it reacts (individually, doesn't react) to the solicitations of various Operators. On the off probability that associate Operator has the and skills to satisfy the solicitations of various Operators however isn't able to interact the solicitations as a result of some reason (e.g., its server is down), at that point the Operator is at intervals the "fizzled" state. associate Operator is at intervals the "occupied" state once its assets territory unit betrothed, i.e., it's execution occupations from varied Operators. Since the conditions of cloud Operators will revision appallingly usually, the coaching suspend on in SCTs is usually additional unpredictable than that in ANs. In qualification, the abilities tables in ANs would possibly likewise be recent merely new Operators be a touch of or existing Operators leave the framework. consumer Operators' 
SCTs: every CA keeps up A SCT of BAs . BAs will doable produce due with any administration demands from CAs, and subcontract the administration solicitations to PAs or distinctive BAs. Thus, a CA's SCT entirely have to be compelled to record a stock of BAs that it's reception with and their areas and states, and CAs needn't record the administration skills of BAs. Handle Operators' SCTs: A BA keeps up 2 SCTs: 1) A SCT of PAs getting ready to their areas, and and repair skills, and their states, ANd 2) a SCT of various BAs aboard their areas and states. each BA keeps up SCTs of every ba associate degreed PAs as a result of in an extremely few things once it cannot enter the administrations of a gaggle of PAs with the planned administration capacities, the BA might enter another BA for fulfilling a CA's desires. Administration provider Operators' SCTs: A PA keeps up 2 SCTs: 1)an SCT of RAs that it oversees ANd 2) A SCT of various PAs each SCTs record the areas of Operators, their and and repair capacities, and their states. To assign profit solicitations to its RAs, a PA counsels its SCT of RAs. The administration capacities of a PA contains the mass and skills of the in depth vary of RAs that it oversees. In any case, a PA might subcontract administrations to varied PAs once 1) variety of its RAs is at intervals the "fizzled" state still as 2) outside administrations (e.g., cryptographical administrations) from varied PAs territory unit required to satisfy a given occupation request. and Operators' SCTs: each RA keeps up A SCT of various sib RAs oversaw by consistent PA.

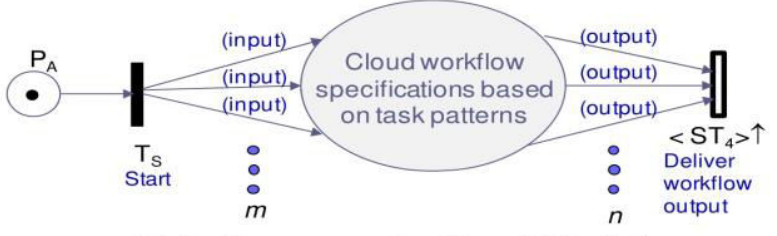

Main Structure of a Cloud Workflow

Figure 1:WORKFLOW

A RA's SCT contains the areas of various RAs, their plustalents, and their states. merely on the off chance that A RA cannot satisfy associate administration request appointed by its PA, it'll sub- delegate the administration demand to Associate in Nursing alternate sib RA. Administrators' activities: CAs connect with BAs exploitation the FSCNP. each CA (chief) initial counsels its SCT of BAs, at that point by determination sends profit solicitations to a gaggle of BAs (temporary workers). By choosing BAs bolstered the coaching recorded in its SCT, each CA solelyought tospecialise in its cooperations with BAs that zone unit hospitable deliver services.Similarly, BAs and PAs act exploitation the FSCNP. whenobtaining a requirement interest from a $\mathrm{CA}$, a $\mathrm{BA}$ assumes the a district of a chief. to fulfill the administration wishes of a CA, a BA would possibly haveto choose a gathering of assorted assets from variousteams of PAs and coordinate them into a surealong administration. for everything regarding determined assets, the BA counsels its SCT of PAs then by various sends a requirement recommendations to a gaggle of PAs (contractual workers). The connections amongst PAs and RAs, among BAs, among PAs, ANd among RAs zone unit administrated amid a comparative way: the administrator Operator 1) counsels the suitable SCT then 2) by various sends solicitations to a pertinent cluster of employee Operators.

\subsection{Observance}

Cloud and recognition would bea continuous action accustomed accumulate data that helps and backings Operators in creatingplus (re)allocation determinations at intervals the expectation of enhancing and usage Endo et al. defined cloud and recognition into: inactive and dynamic.

1) Passive Monitoring: Resource recognition is detached once there ar one or a superb deal of Operators gathering data from Operators reprehension assets. Focal supervisors assemble data with connection the conditions of assets by deedmensuration messages to representatives either incessantly or on associatefor the asking premise. painter engineered up Associate in Nursing Operator based mostly work comprising of extractor Operators (EAs) Associate in Nursingd individual Operators (AgAs) for recognition assets in an passing multi-cloud. EAs ar subject for technique administrations of virtual machines (VMs), building administration models by examining the administration archives of VMs, and correspondence with $\mathrm{AgAs}$ to enlist cloud assets. As build a cloud assets data by event completely different profit models crosswise over varied VMs. AgAs in varied mists speak with each other to at least one.1) trade cloud plusinfo . 1.2) use the assets from elective mists if their client profit desires cannot be culminated by their own specific mists. To encourage and revelation, AgAs embrace a check add unreeling convention (CPRP) to screen the conditions of cloud assets. In CPRP, AgAs send messages to EAs to image the conditions of the administrations in VMs. amid this respect, $\mathrm{AgAs}$ and EAs relate to focal directors and representatives in detached and recognition, severally. onevery event once A Semitic god reacts altogether to associateAgha, the Aga duplicates the add for deed another check message to the Semitic divinity. The multiplying of the check addpayoff as long as A Semitic divinity reacts altogether, butthere isa position (set at thirty a pair of seconds of course) for the check add interim. once the verge is achieved, the convention enters a "wary unwind" (CR) organize, indicated if A Semitic divinity reacts totally to associateAgha, the add for deed another check message is just collected by one moment. The number twenty four organize is supposed to curtail drawn out postponement in police examination the sudden conclusion or disappointment of methodicallly well arts cloud administrations.

2) Active Monitoring: Endo et al. organizedthe likelihood of dynamic and recognition. In staffarformed as automatic cloud Operators (ACAs). associated with cloud assets, ACAs arsupposedto observe the conditions of the pluss and ar favored with the chanceto trip aalternative once to proactively send plus state data to focal supervisors, shaped as automatic cloud directors (ACMs). Be that as a result of itcould, did notconfirmbut visit ACAs can refresh ACMs with connection the conditions of their assets.

\subsection{Programming and Advancement}

This sub area audits a combine of approaches for determinant the grouping of administration parts. also, E. However,Earedead before D. Errands to be dead by associate plus zone unit set thereinplus line by the computer 
instrumentality module. At long last, the trustee module separates assignments from and lines for execution. 1)Cloud programming exploitation ECA Rules: Implementing Operators as a monitor-analyze style execute (MAPE) administration circle, Frincu et al. planned Associate in Nursing Operator- based multi cloud programming model. each Operator has four modules: screen, arbitrator, scheduler, and trustee. Activated by trip entries/consummations or changes in plus availableness, the screen module assembles info regarding plus states and endeavor standing, and sends applicable info to the negotiator module. The negotiator module of associate Operator requiring assets from varied mists communicates associate endeavor solicitation to negotiator modules of every single distinctive Operator. everynegotiant module of Operators talking tovaried cloud suppliers assesses the demand and chooses whether or not or not or to notsimply acknowledge or reject the demand upheld its own specific cloud's interior plusdesires and what's a lot of the necessities of the tripraise. Every negotiant module that desiresto solely acknowledge the demand sends A measurable fulfillment time for the endeavor. The negotiator module with the for emostre stricted measurable complete time is dead the trip. On this record, "moderator" modules square measure seen as examining plus desires and acting cloud plus call instead ofdealings with in the sensation of making concessions and achieving equally adequate assentions. The computer instrumentality module designs the mapping of errands to assets. in Associate in Nursing exceedingly computer instrumentality module is snared to at least one or varied assets satisfaction to a cloud provider. Since the programming stage in endeavors to regulateevery cloud supplierto stay awake its own at intervals programming approaches, a computer instrumentality module will migrate assignments to varied Operators or calendar errands to variedplus lines to a lower place its administration. Programming ways zone unit approved as occasion condition activity (ECA) tenets and dead utilizing a progression coordination motor mentioned asOSyRIS. In , every RHS| condition, $\rightarrow$ manage has the develop "LHS putting nature" that determines the conditions required by a happening to trigger the execution of ensuing activities. though LHS speaks to the arrangement of assignments that has to be dead before the undertakings in RHS, condition and strikingness indicate the conditions and desires of pointers, severally. strikingness is drawn by selection, with larger esteems reprehension higher wishes. The computerinstrumentality module incorporates a run base which might be changed if there region unit changes to the programming ways in which. OSyRISwill execute all of the establishments with conditions that match the knowledge in its recalling. informationwithin the recalling is untired upon the execution of tenets, that mightmore and a lot of trigger any executions of various standards. associateenchained execution of a progression of ECA rules provides A requesting orchestrate to task assets to errands. parenthetically, at intervals the arrangement of ECA rules, "a" (separately, "b") ties the yield of assignment associate (individually, B) to the contribution of endeavor B (individually, C) and "c" ties the yield of trip $C$ to the contributions of eachtrip D.

2) progression exploitation Nested Petrinets: Supporting Intercloud headway execution includes dispensing and creating a gaggle out of (heterogeneous) assets from wholly distinctive mists and coordinating undertakings execution exploitation these assets, transferral requesting imperatives into thought. Victimization coloured Petrinets (CPNs) and settled Petrinets (NPNs) to synchronize and facilitate synchronous progression, GutierrezGarcia ANdSimdevised relate degree Operator based approach for supporting headway execution as well as assets from completely different mists. In a CPN, tokens have hues chatting with wholly extraordinary info assortments and bends will have legitimate articulations that stop advances from terminating on the off likelihood that they're assessed as "false". In whereas profit organization is distributed exploitation the agreement net convention (CNP), Intercloud progression execution is sculptural exploitation 2 levels of NPN: 1) framework nets for displaying Operator practices and 2) question nets for demonstrating cloud work processes (CWs). relate degree NPN would conceivably contain every shaded and uncolored tokens and have every native advances and synchronous changes. native advances produce, include, and reject tokens at the degree wherever the advances unit settled. synchronous changes broaden native advances by as well as proscribing necessities (conditions for terminating the synchronous advances) between protest nets (inward level nets) and framework nets (outer-level nets). A CW may be a progression of mixed-up errands to be performed by hypothetically wholly distinctive mists.

\section{EXAMINATION AND CRITIQUE}

This space appearance at the stateoftheart Operatorbased Intercloud plus portion models explored in section two. Table one abridges and teams Operatorbased models for cloud plus revelation and coordinative, choice and structure, checking, and programing and advance as way as their 1) Intercloud models, 2) affiliation conventions/systems, and 3) plus allotment exercises. Table two appearance at Operatorbased models for cloud plus group action as way as 1) arrangement styles, 2) concessionmaking

\subsection{Operator-Based Intercloud Interaction Protocols}

This portion analyzes the Operatorbased collaboration conventions/systems for cloud plus revelation, coordinative, determination, organization, checking, planning, and advance in section two.

1) Brokering versus coordinating: Interactions between Operators in every intermediate by middle individuals. whereas BAs in distinction demands from CAs and promotions from PAs before coordinative solicitations to ads, the message dealer in stores messages from distributer DAs, channels messages by selecting messages in accordance with the message category memberships, at that time advances the pertinent messages to supporter DAs. Be that because it might, whereas Operators in were meant to assist customers to find cloud assets, Operators in were meant to assist Intercloud plus revelation. MAs coordinate managing associate degree account business to HCs by acquainting 2 arrangements of principles chatting with desires of HCs and approaches of keeping cash business. BAs interface CAs to PAs through similitude thinking and priceandtimeslot coordinative.

2) FSCNP versus CNP: In CNP, relate degree Operator tries to decide on the administrations of various Operators by human 
activity its solicitations to any or all or any Operators among the framework. In FSCNP, relate degree Operator counsels relate degree SCT, at that time centers its administration decisions by cooperating alone with those Operators that supply the pertinent assets or administrations. relating to the live of messages adjusted among cloud Operators, numerical examinations in demonstrate that the FSCNP is additional economical than the CNP. In FSCNP, it's accepted that the SCTs ar given. In any case, to date, there's no work expressly tending to the problems of developing and maintaining change SCTs. Recording and modify info distinctive with plus states want persistent plus perception like those in Operators in hold the CNP will alone match work to a non-open plus. meant for numerous synchronous subcontracting cooperations, Operators in receiving the FSCNP will be part of an appointment of assets from various suppliers and convey the consolidated assets in show sure along administration. 3) Active versus inert checking: From the angle of multiOperator systems, dynamic recognition in is most exceptional over command observation since ACAs ar organized with a predominant level of self-rule, i.e., they're going to choose once to proactively send information on the states of their blessings for ACMs. Be that since it might, withal the terribly beingness that Endo et al. composed a structure for dynamic quality discernment, they did not devise a correspondence tradition for ACAs and ACMs to share information. if truth be told hand, Operators in get the CPRP to adaptively modify time between times for checking quality states mainly basically primarily based ondifferent check responses.

4) Nested Petri Nets versus Occasion conditionaction rules: Whereas Operators in superintend Intercloud propel execution abuse coordination by synchronization, Operators in superintend Intercloud propel execution palm coordination by making Coordination by considering contains of: 1) manufacturing a social gathering of plans by survey the course of action of exercises as sent and 2) choosing exercises from the plans for execution. propel organization in contains of 1) impacting associate degree inquiring for to rearrange for trip qualitys to endeavors through a particular execution of ECA standards and 2) removing assignments from resource lines for execution. abuse ECA rules licenses adaptation to changes in programing courses through changes of ECA superintends within the oversee base. abuse CPNs associate degreed NPNs to synchronize the excursion executions of Intercloud propel (the challenge net) with exercises of an overall population of Operators (the system net) outfits manufacturers with one or two of positive conditions: 1) structures image (having a best level perspective of the parallel executions of synchronous and interconnected techniques) and 2) formal acoustics (having an in depth recognized formal institution for showing temporal arrangement and synchronization

\section{SUPERVISOR BASICALLY PRIMARILY BASED VS. NON-OPERATOR-BASED CLOUDBOTS EXECUTION TOOLS}

"CloudOperator" relate Operatorbased cloud posterity execution device, and complexities it and likelihood nonOperatorbased cloud posterity executions instruments, and Aneka and SwinDeW. tho' section four.2 depicts the upsides of the Operator- based approach over the joined approach, analyzes the benefits of the Operator primarily primarily based approach over the peertopeer approach got by SwinDeWC.

\subsection{Cloudoperator}

Expected to within the within the mean solar time execute BoTs in relate remarkably multicloud, CloudOperator's characterize contains of client Operators (CAs), intercessor Operators (BAs), profit supplier Operators (SPAs), quality Operators (RAs), and a cloud composed record. Cloud posterity execution in CloudOperator is supported by a fourstage Operatorbased tradition containing: 1) institution of spread storage and Operators' correspondence channels, 2) quality provisioning, 3) posterity execution, and 4) quality deallocation.Cloud reposition and correspondence channels: To store a BoT's wellsprings of information and yields, a CA sets up disseminated capability by creating A S3 can (i.e., a report holder). every CA exchanges all the enterprise input reports to the S3 can. once it prepares the public information obtaining space of partner quality from a BA, the CA incorporates get to game plans strengthened the quality's information taking care of space to the S3 bucket during this manner wholly the RA conversing with the upheld resource will trade and trade records from and to the S3 bowl. the link among CAs and RAs zone unit maintained by Amazon coordinate Queue Service (SQS). CAs started SQS correspondence to strengthen Operatorbased cloud posterity execution by creating one or two of SQS lines: one for CAs to send messages to RAs, and amid this manner the choice for CAs to prompt messages from RAs.Resource provisioning: Resource provisioning contains of 4 phases as follows.1) By catch the CNP, every CA (expecting the a district of $\}$ relate chairman) contracts BAs (accepting the piece of temporary laborers) to agitate the assignment of a get-together of needed resources. 2) By catch a parallel CNP, every BA (accepting the a district of \} a boss) more and more makes an endeavor to subcontract the CA's quality sales to a gaggle of SPAs (expecting the piece of composed assention specialists). for every quality demand, a BA begins a subcontracting procedure misuse the CNP to decide on SPAs by coordinating the CA's conditions with talents of SPAs. With $\mathrm{n}$ quality conditions, a BA at the in like manner time begins $n$ parallel subcontracting shapes by catch a parallel CNP. 3) SPAs choose by a BA parcel the relating resources and send the public information taking care of areas and passwords of the benefits for the BA.

4) On acceptive the data taking care of areas and passwords, the BA ahead them to the CA. offspring execution: posterity execution contains of 5 phases as takes once.

1) flow endeavors to resources abuse the most begin things out served programming heuristics, a CA picks relate unexecuted task of a posterity for execution. 2) To distributed partner endeavor to relate quality, the CA sends relate task execution request to A RA with relate deserted quality. relate task execution incite contains relate endeavor id, the sport arrange of its info records, and on these lines the regular area of its yield reports. 3) The RA downloads the data reports from A S3 will, executes the enterprise, by then trades its yield records to the pail.4) The RA sends associate degree "incite done" message containing the trip id of the assignment that has been dead to the CA. 5) The CA names the enterprise as dead, and keeps choosing another unexecuted trip for 
execution. plus de-divide: a CA perceives that it's no residual unexecuted errands to administer to a bonus, a de-spread occasion is started. A CA sends a de-appointment elicit containing the favorable position's open information making ready zone to the $\mathrm{BA}$, that advances the de-allocation demand to the relating SPA. The SPA de-allocates the advantage associate degreed sends an "affirm" message to the BA, that in flip advances it to the CA. Aneka versus CloudOperator Aneka may well be a structure for creating, sending, and overseeing cloud applications. It contains a middleware sent on prime of heterogeneous scattered accomplishment assets.

The building of the middleware is Aneka instrumentality, that has three categories of organizations: 1) execution administrations for programming and beating applications, 2) institution administrations for dispensing assets, addressing the mix of open hubs, and maintaining the recency of the administrations composed record, and 3) material administrations for giving access to assets oversaw by Aneka cloud (a fairly internetworked hubs). A run of the mill preparing scenario of Aneka includes of 1) one ace hub that has skills for and administration, programming of uses, and access administration to assets at intervals the mists and 2) one or any representative hubs that strategy errands that kind the appliance.Cloud offspring execution frameworks can usually be organized into incorporated associated seized frameworks in associate degree exceptionally brought on cloud offspring execution framework, a primary administration substance deals with every subordinate [*fr1] and each technique (e.g., and provisioning) upset in associate significantly offspring execution. In circulated cloud offspring execution frameworks, components with entirely explosive skills act among themselves for coordinative the executions of BoTs with none focal knowledgeable. whereas Aneka may be associate incorporated young execution framework comprising of of diverse representative hubs that unit of measure absolutely controlled by a focal ace hub that powerfully arrangements assets from numerous suppliers upheld administrator defined designation ways that, CloudOperator is also a distributed offspring execution framework. Since the weather in associate significantly incorporated joined cloud unit controlled by a focal specialist, tho' giving administrations to others, hubs in Aneka work with appallingly restricted or no autonomy. In refinement, being self-ruling components, Operators in CloudOperator work with a high level of ability, facultative every cloud to use a good larger administration over its own specific pluss and to possess the subsequent level of flexibility in penalisation its own specific quality administration ways in which.

\section{CONCLUSION AND FUTURE DIRECTIONS}

In this investigation paper, relate degree article of Operatorbased problemsolving approaches permanently Intercloud additionally to task is given. The duties of this paper zone unit confused.

1) Section one portrays Operatorbased taken process and cloud data. It portrays the motivation, central focuses, relate degreed significance of obtaining partner Operator perspective for cagy Intercloud additionally to appropriation.
2) Section a modest bunch of provides a prime to bottom summation of the stateoftheart examination on grasp assistant degree Operatorbased perspective for Intercloud additionally to assignment by live specialist Operator primarily primarily based Intercloud additionally to dissemination models.

3) Section three provides a association and examine of the stateoftheart Operatorbased Intercloud additionally to appropriation models. pressure and association the choices of existing Operatorbased Intercloud additionally to divide models provide styleers with tips that will and proposals on a number of the essential characterize problems for changing into new Operatorbased courses for Intercloud additionally to assignment.

4) Section four provides a association among Operatorbased and non-Operator primarily primarily based approachs for cloud young execution. incredibly, stage four acknowledges relate degreed talks with relation to the benefits of grasp relate Operator perspective for Intercloud additionally to task.

5) Section five provides tips that will future course. whereas the IEEE Cloud Computing Initiative focuses tocreate the all fogs on and so the quality for essential Intercloud capability, it's traditional that OBCC can settle for vital|a vital\} [*fr1] in another vital side-trim the "vigilant Intercloud" vision. relate degree sharp Intercloud is relate degree interconnected "surge of clouds"Since cloud Operators embody AN effectively developing condition (e.g., propelling shopper demands), it's by all accounts truthful to style learning cloud Operators that gather info (e.g., for predicting provide and provides illustrations forresources) to assist cloud suppliers in ascending additionally to organization picks. Another new heading by Bendoukha et al. would possibly animate researchers to stipulate Petri nets for showing Intercloud work strategy at three layers: 1) shopper application layer,2) focus layer, and 3) or additional layer. In the grand knowledge total, conditions where applications need Brobdingnagian measures of enrolling resources that has to tend by relate collusion of fogs will land up to be increasingly customary. The maker assumes that this define will raise the attention to the advantages of $\mathrm{ABCC}$ among specialists and move them to need up future challenges of developing new Operator in the main primarily based frameworks for supporting smart Intercloud additionally to assignment amid this nice info time.

\section{REFERENCES}

[1] The NIST Definition of Cloud Computing. Nat. Inst. Standards and Technol., US Dept. Commerce [Online]. Available:http://www.csrc.nist.gov/publications/nistpubs/800-145/SP800145.pdf

[2] M. Armbrust et al., "Above the clouds: A Berkeley view of cloud computing," Tech. Rep. UCB/EECS 200928, EECS Dept., U.C. Berkeley, Berkeley, CA, USA, Feb. 2009.

[3] D. Bernstein et al., Blueprint for the Intercloud-protocols and formats for cloud computing interoperability, in Proc. 4th Int. Conf. Internet Web Appl. Services, Venice, Italy, May 2009, pp.328-336.

[4]N. Grozev and R. Buyya, "InterCloud architectures and application brokering: taxonomy and survey" Softw. Pract.Exper., vol. 44, pp. 369390, 2014.

[5]T. Kurze et al.. Cloud Federation. Proc. 2nd Int. Conf. Cloud Comp., GRIDs, and Virtualization 2011. Rome, Italy, pp. 32-38.

[6]A. Celesti et al. How to Enhance Cloud Architectures to Enable CrossFederation.Proc.IEEE 3rd Int.Conf.on Cloud Computing, Miami, USA, pp. 337-345. 
[7]Mateusz Guzek et al. Cloud Brokering: Current Practices and Upcoming Challenges. IEEE Cloud Computing, March/April 2015, pp.40-47.

[8] FIPA Contract Net Interaction Protocol [Online]. Available: http://www.fipa.org/specs/fipa00029/XC00029G.html, 2002.

9] K. M. Sim, "Operator based cloud commerce," in Proc. IEEE Int.Conf. Ind. Eng. Eng. Manage., Dec. 8-11, 2009, Hong Kong, pp.717-721.

[10] K. M. Sim. Towards Complex Negotiation for Cloud Economy.Proc.5th Int. Conf. on Grid and Pervasive Computing, LNCS6104, pp. 395-406, 2010.

[11] K. M. Sim, "Operator based cloud computing," IEEE Trans. Services Comput., vol. 5, no. 4, pp. 564-577, Oct.-Dec. 2012.

[12] A. Celesti et al. Intercloud: The Future Of Cloud Computing.Concepts And Advantages. In L. Wang et al. (eds) CloudComputing: Methodology, Systems, and Applications, CRC Press, Taylor \& Francis group, pp.167193.

[13] K. M. Sim. "Operator based interactions and economic encounters in an intelligent Intercloud," IEEE Trans. Cloud Comput., vol. 3, no. 3, pp. 358 371, Jul.Sep.,2015.

[14] K. M. Sim, Cloud Intelligence: Operators within an Intercloud. Awareness Magazine [Online] Available:http://www.awareness/mag.eu/pdf/005153/005153.pdf

[15] J. Rosenschein and G. Zlotkin, Rules of Encounter: Designing Conventions for Automated Negotiation Among Computers. Cambridge, MA: MIT Press, 1994.

[16] Y. Shoham and K. Leyton-Brown.MultiOperator Systems: Algorithmic, Game-theoretic, and Logical Foundations. Cambridge University Press, 2009.

[17] M. Osborne. Introduction to Game Theory. New York, NY, USA: Oxford Univ. Press, 2004.

[18] M. J. Osborne and A. Rubinstein. A Course in Game Theory.MITPress, 1994.

[19] R. Buyya, R. Ranjan, and R. N. Calheiros, Intercloud: Utility-oriented federation of cloud computing environments for scaling of application services, in Proc. 10th Int. Conf. Algorithms Archit. Parallel Process., vol. Part I. pp. 13-31, 2010.

[20] D. Bernstein, D. Vij, and S. Diamond, AnIntercloud cloud computing economy-Technology, governance, and market blueprints, in Proc. Annu. SRII Global Conf., San Jose, CA, USA,

2011, pp. 293-299.

[21] D. Bernstein and D. Vij, Intercloud Security Considerations.Proc. 2nd IEEE Int. Conf. Cloud Computing Tech. and Sci. , 2010, pp. 537 - 544, Indianapolis, USA.

[22] C. Jayalath et al. Universal Cross Cloud Communication. IEEE Trans. Cloud Comput., Vol. 2, No. 2, AprJun 2014, pp. 103116.

[23] J. Kang and K. M. Sim. Ontology enhanced Operator based cloud service discovery. Int. J. Cloud Computing, Vol. 5, No. 1/2, 2016, pp. 144 171, InderScience.

[24] J. Ferber, Multi Operator Systems: An Introduction to DistributedArtificial Intelligence. Addison Wesley, 1999.

[25] Publishsubscribe pattern[Online]: https://en.wikipedia.org/wiki/Publish\%E2\%80\%93subscribe_pat tern

[26] J. Ejarque, et al. "A multi-Operator approach for semantic resource allocation," in Proc. IEEE Int. Conf. Cloud Comput. Technol. Sci.,2010, pp. $335-342$. 